Revue de droit comparé du travail et de la sécurité sociale

2| 2018

Harcèlement moral au travail en jurisprudence

comparée

\title{
La jurisprudence russe sur le harcèlement moral au travail
}

\section{Elena Sychenko}

\section{(2) OpenEdition}

Journals

Édition électronique

URL : https://journals.openedition.org/rdctss/1951

DOI : $10.4000 /$ rdctss. 1951

ISSN : 2262-9815

Éditeur

Centre de droit comparé du travail et de la sécurité sociale

Édition imprimée

Date de publication : 1 juin 2018

Pagination : 78-85

ISSN : $2117-4350$

Référence électronique

Elena Sychenko, « La jurisprudence russe sur le harcèlement moral au travail », Revue de droit comparé du travail et de la sécurité sociale [En ligne], 2 | 2018, mis en ligne le 01 novembre 2021, consulté le 13 novembre 2021. URL : http://journals.openedition.org/rdctss/1951 ; DOI : https://doi.org/10.4000/ rdctss.1951

\section{(c) (i) $\ominus$}

Revue de droit comparé du travail et de la sécurité sociale est mise à disposition selon les termes de la Licence Creative Commons Attribution - Pas d'Utilisation Commerciale - Pas de Modification 4.0 International. 


\section{LA JURISPRUDENCE RUSSE SUR LE HARCÈLEMENT MORALAU TRAVAIL}

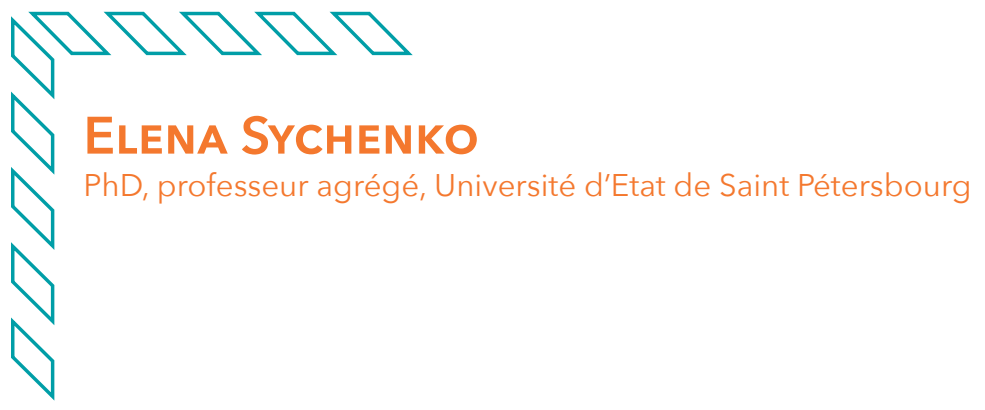

L

e risque de harcèlement moral (intimidation) est considéré comme l'un des principaux défis par les spécialistes de la santé et de la sécurité au travail et les décideurs en Europe où un certain nombre de mesures ont été mises en œuvre. Ces mesures incluent des directives européennes établissant la possibilité de traiter le harcèlement comme une discrimination, des instruments juridiques non contraignants sur le harcèlement, des législations nationales et de la jurisprudence. En Russie, cependant, la notion de harcèlement moral au travail n'est pas reconnue par le droit du travail et les spécialistes soulignent la nécessité d'adopter des dispositions spéciales sur ces questions ${ }^{1}$. Pourtant, l'absence de telles dispositions ne signifie pas l'inexistence de tels phénomènes ou de demandes des salariés de protéger leur dignité et leur intégrité morale à l'aide de normes légales, y compris par des normes non conçues à cet effet. Dans cette contribution, nous examinerons la jurisprudence portant sur le harcèlement moral au travail. Une première partie sera consacrée aux actes qui pourraient être qualifiés de harcèlement moral (I). En l'absence de toute définition juridique en Russie, il nous faut mettre en évidence une référence pour expliquer le choix des décisions de justice retenues pour cet article. Dans une deuxième partie, plusieurs jugements concernant le harcèlement moral seront examinées (II). Les hypothèses d'une 'éventuelle application de la Convention européenne des droits de l'homme pour la protection contre le harcèlement sur le lieu de travail seront présentées dans une troisième partie (III).

1 A. Lushnikov, « Pravo rabotnika na ohranu truda: novye podhody (Le droit du travailleur à la santé et à la sécurité au travail: nouvelles approches) ", Sotsial'noye i pensionnoye parvo, 2009, n 52, p. 1. 


\section{I - DÉFINITION ET BREF EXAMEN DE LA LÉGISLATION RUSSE}

En Russie, la nécessité de protéger la dignité des travailleurs a été reconnue comme I'un des principes fondamentaux du droit du travail dans le Code du travail adopté en 2001, même s'il est difficile de dire que ce principe ait été appliqué dans la pratique. Ces dernières années, de nombreux articles de recherche sur ce problème ont été publiés, en adoptant un point de vue psychologique ou juridique ${ }^{2}$. II a été noté qu'entre $5 \%$ et $20 \%$ des salariés russes ont été victimes de harcèlement moral ${ }^{3}$.

L'article 2 du Code du travail russe prévoit le droit des salariés à la protection de leur dignité. Il convient de noter que dans tous les cas examinés ci-après, la Cour ou le requérant se sont référés à ce principe. Une autre norme importante figure à l'article 237 du Code du travail russe. Elle prévoit l'obligation de l'employeur d'indemniser le préjudice moral en cas d'action illégale ou en l'absence de mesures appropriées prises à l'égard du salarié. Il sert de cadre pour l'indemnisation des dommages et a également été mentionné dans toutes les affaires ici relevées.

L'invocation de l'interdiction des discriminations, fixée dans l'article 3 du Code du travail, est une nouvelle tendance dans les affaires de harcèlement moral. Les requérants considèrent que la persécution de la part de l'employeur constitue une discrimination, mais ne parviennent pas à le prouver, compte tenu des normes très restrictives de la procédure civile.

Pour choisir les affaires à relater dans cette contribution, nous avons tenu compte de la définition du harcèlement moral formulée par le législateur français et considéré comme tels les actes répétés visant ou conduisant à une détérioration des conditions de travail qui porteraient atteinte au droit à la dignité et l'intégrité physique ou mentale des victimes ou compromettraient l'avenir professionnel du salarié.

\section{II - LA JURISPRUDENCE SUR LE HARCÈLEMENT MORAL EN RUSSIE}

Il convient de noter d'emblée qu'il n'existe pas de statistiques judiciaires officielles sur les affaires impliquant le droit du salarié à la protection de sa dignité, les jugements auxquels nous ferons référence n'ayant jamais fait l'objet d'une analyse juridique ce qui démontre que l'intérêt suscité par ce sujet est plutôt limité.

La recherche des jugements a été effectuée à l'aide d'une recherche par mot clé dans les bases de données des décisions rendues par les tribunaux russes. Les mots clés étaient les suivants : «harcèlement » (travlya), « insulte » (oskorblenie), " persécution » (presledovanie), «intimidation ». Il est intéressant de noter que nous avons trouvé deux cas dans lesquels le demandeur a fait référence à la notion de harcèlement et d'intimidation, ce qui démontre que le niveau de connaissance des salariés dans ce domaine augmente lentement ${ }^{4}$. Dans l'une des affaires, un tribunal russe a invoqué le principe de la protection de la dignité du

2 Voir, par exemple, N. «Plastinina, Kak zashchishchat'sya ot mobbing (Comment assurer la protection contre le harcèlement?)», Trudovoye pravo, 2012, p. 2.

3 G. Bryntseva, « Mobbing Dik», Rossiyskaya gazeta, 24 mars 2010.

http: //www.rg.ru/2010/03/24/mobbing.html

4 Décision n 2-112/2017, 23 janvier 2017, Tribunal du district de Leninsky de Nijni Taguil (Région de Sverdlovsk). 
salarié et l'a interprété comme mettant à la charge de l'employeur l'obligation d'indemniser le préjudice moral dans le cas où le salarié subit un traitement dégradant 5 .

Les chercheurs qui ont étudié ce sujet en 2014 ont mis en lumière le fait que les affaires traitant de la question du harcèlement moral se rapportent à la discrimination, au licenciement abusif, ou aux deux, mais ne visent pas à lutter contre le harcèlement ou le harcèlement moral proprement dit $^{t}$. Cette observation est toujours pertinente, mais devrait être complétée par les cas de sanction disciplinaire illégale. En premier lieu, nous examinerons les cas où le requérant a fait état de persécutions de la part de l'employeur qui ont pris la forme d'une discrimination concernant les conditions de travail et le paiement des salaires (A). Ensuite, nous envisagerons les cas de licenciement abusif (B) et les sanctions disciplinaires injustes (). Enfin, seront examinés quelques cas de sanctions disciplinaires infligées pour avoir commis des actes de harcèlement (C).

\section{A - CONDITIONS DE TRAVAIL INJUSTES CONSTITUANT UNE FORME DE PERSÉCUTION}

Un certain nombre d'affaires concernaient une forme de persécution telle que le non-paiement de primes que le salarié avait l'habitude de recevoir. Généralement, il n'y a pas d'obligation contractuelle d'effectuer de tels paiements et cela reste entièrement à la discrétion de l'employeur. Dans un des cas, la requérante a réussi à prouver que le nonpaiement des primes et la pression psychologique exercée sur elle après avoir porté plainte devant l'inspection du travail constituaient une discrimination ${ }^{7}$. Elle a reçu des dommagesintérêts pour préjudice moral d'un montant d'environ 40 euros (3 000 roubles).

Dans deux autres affaires, les tribunaux n'ont pas pris en compte les arguments des requérants sur le conflit avec l'employeur et les pressions exercées sur eux pour qu'ils quittent leur emploi et ont examiné l'affaire uniquement à la lumière du droit de l'employeur de diriger ses salariés. Ainsi, les deux demandes ont été rejetées ${ }^{8}$.

Dans l'une des affaires', l'employeur a refusé de fournir un contrat écrit à la salariée, une gardienne dans une colonie de vacances, et a commencé à la persécuter après qu'elle lui ait demandé de formaliser la relation de travail en cours. Cette persécution a pris la forme de réductions de salaire et il lui a été interdit d'utiliser la loge des gardiens quand elle travaillait. Elle a demandé au tribunal de reconnaître l'existence de la relation de travail et d'ordonner le paiement du préjudice moral. Le tribunal a constaté la violation du droit du travail, cependant, les circonstances du harcèlement n'ont pas été prises en compte dans les conclusions du tribunal, qui n'a accordé son attention qu'à la partie formelle de l'affaire,

5 Décision en appel du tribunal régional de Tomsk n 33-4101/2011, du 30 décembre 2011.

6 D. Chernyaeva, "The Regulation of Mobbing and Harassment in Russia: Current Challenges and Future Prospect, 14-31 », E-Journal of International and Comparative Labour Studies, 2014, n 3, p. 2.

7 Décision n² 2-307/2012, March 28, 2012, tribunal du district Oktyabrsky de Saransk (République de Mordovie).

8 Décision $n^{\circ}$ 2-16242/2016, 10 février 2017, tribunal de Surgut; Décision n² 2-138/2016, 15 mars 2016 dans l'affaire $n^{\circ} 2-138 / 2016$, tribunal municipal de Kimovsky (région de Toula).

9 Décision n 2-1331/2011, 21 Décembre 2011, tribunal de district d'Irkoutsk, des circonstances similaires existaient dans l'affaire $n^{\circ}$ 2-174/2013, 22 Mars 2013, tribunal de district Zaeltsovsky de Novossibirsk. 
à savoir l'absence de contrat écrit.

\section{B - ACTES DE HARCÈlEMENT DANS LES AFFAIRES DE LICENCIEMENT ABUSIF}

Selon la législation du travail russe, l'employeur peut licencier le salarié pour les motifs énumérés dans la loi. Dans un certain nombre de cas, le salarié a subi un harcèlement ayant pour but de lui faire quitter volontairement son emploi. La Cour suprême a souligné que dans les cas où le salarié allègue qu'il a été forcé de démissionner, les tribunaux inférieurs doivent examiner si la démission était volontaire ${ }^{10}$, le fardeau de la preuve incombant au salarié.

Les décisions en faveur du requérant reconnaissant que le salarié a été forcé de démissionner en raison d'un harcèlement moral sur le lieu de travail sont presque inexistantes. Toutefois, dans une affaire, le tribunal a décidé que le salarié avait été forcé de démissionner à la suite de la création d'un environnement hostile par l'employeur, et que la démission du demandeur était le résultat de facteurs émotionnels négatifs ${ }^{11}$. Dans la plupart des cas, les requérants souffrant d'un environnement de travail hostile n'ont pas réussi à obtenir gain de cause car ils n'ont pas réussi à prouver la coercition ${ }^{12}$. Dans de nombreux cas, les requérants alléguaient que le licenciement disciplinaire ou le licenciement dans le cadre d'un plan social étaient la dernière étape de leur harcèlement et constituaient la dernière partie de la guerre menée contre les salariés. La Cour a abordé toutes ces affaires de manière très similaire. Elle a limité l'analyse à l'examen de la procédure de licenciement sans examiner les allégations de harcèlement (sanctions disciplinaires antérieures multiples, réductions de salaire, pressions psychologiques).

Dans une affaire de licenciement abusif pour multiple violations des devoirs de la salariée ${ }^{13}$, la requérante a allégué que la direction avait décidé « d'organiser un harcèlement à son encontre », en contrôlant chaque étape de son travail et en faisant évaluer son travail par une commission dépourvue de spécialistes de son domaine d'activité. Elle estimait qu'une telle commission avait été créée pour exercer une pression psychologique sur elle, elle s'était vu confier des tâches qui n'étaient pas vraiment réalisables et qui ne faisaient pas partie des tâches correspondant à son poste. Le tribunal a jugé que l'employeur a le droit de contrôler la qualité du travail, y compris au moyen de l'établissement de commissions. C'est pourquoi, pour citer les termes du jugement, « le tribunal considère que les arguments de la requérante sur la pression psychologique sont sans fondement ». Cette citation est digne d'attention car elle témoigne de l'approche générale des tribunaux vis-à-vis de ce type de cas, c'est-à-dire qu'elle est très formelle et superficielle. Elle examine uniquement la conformité de la procédure de licenciement avec les normes pertinentes sans se demander si cette procédure a été effectuée d'une manière équivalant à un traitement dégradant ou était le résultat d'un tel traitement.

10 Décision en session plénière de la Cour suprême sur l'application du Code du travail de la Fédération de Russie par les tribunaux de la Fédération de Russie (adoptée le 17 mars 2004).

11 Décision de la Cour de district du district central de Tchita n² 2 -1055-07, 3 mai 2007.

12 Jugement en cassation $n^{\circ}$ 33-4995 du tribunal régional de Perm, adopté le 23 mai 2011.

13 Décision $n^{\circ}$ 2-112/2017, 23 janvier 2017, Tribunal de district Leninsky de Nizhny Tagil (région de Sverdlovsk). 
Dans une autre affaire, un salarié a été licencié pour plusieurs manquements à ses fonctions après avoir signalé la qualité insuffisante du travail de son service. L'employeur a commencé à le persécuter, en modifiant son temps de travail et en ne fournissant pas de conditions de travail appropriées ainsi qu'au moyen de sanctions disciplinaires illégales. La Cour a conclu que la procédure de licenciement violait le Code du travail et a ordonné la réintégration, le préjudice moral étant estimé à un montant de 15 euros ( 1000 roubles) ${ }^{14}$.

II y a eu plusieurs affaires relatives à un harcèlement présédant des procédures de licenciement. Dans l'une d'elles ${ }^{15}$, la requérante qui était employée municipale a allégué que l'employeur avait "organisé une persécution intentionnelle afin de la "forcer » à quitter son emploi ", qu'elle était victime de discrimination et était traitée d'une manière dédaigneuse dans son environnement de travail. La Cour n'a pas examiné son allégation de traitement dégradant. Elle a examiné les procédures de licenciement et a conclu que l'employeur n'avait pas respecté les règles, et a réintégré la requérante en ordonnant le paiement de 40 euros à titre de réparation du préjudice moral.

Une autre affaire ${ }^{16}$ de licenciement a été considérée de la même manière formelle. Bien que la requérante ait fait valoir que le fait de la choisir au moment de la licencier était le résultat de la persécution de l'employeur à son égard, la Cour a déclaré que «les motifs qui guident l'employeur lorsqu'il s'agit de réduire le personnel n'ont pas d'importance juridique et ne peuvent être examinés par la justice ».

Dans une autre affaire ${ }^{17}$, la requérante a déclaré qu'elle était considérée comme licenciée, l'employeur lui ayant « déclaré la guerre » après qu'elle ait refusé de travailler sans rémunération. Après cela, elle a été persécutée par l'employeur qui lui a retiré une partie de ses fonctions, et a augmenté la quantité de travail, a pris le contrôle de l'ordinateur sur lequel travaillait la demanderesse et sur lequel elle ne pouvait plus ouvrir de documents ou sauvegarder des dossiers. La connexion Internet a été désactivée. Le tribunal a rejeté sa demande car elle n'a pas réussi à prouver ces actes. La législation russe ne prévoit pas d'inversion de la charge de la preuve dans les cas de discrimination et il est évident que dans les cas où le salarié doit prouver certains actes qui n'ont pas été documentés, il est très probable qu'il n'y arrive pas.

Pour résumer les jugements ci-avant étudiés, il convient de noter que les termes du tribunal selon lesquels « les motifs qui guident l'employeur lorsqu'il décide de réduire le personnel n'ont pas d'importance juridique et ne peuvent être soumis à un contrôle judiciaire "sont la clé pour comprendre les problèmes posés par la protection contre le harcèlement. Les actes de harcèlement en tant que tels ne sont perçus comme une violation des droits que s'ils atteignent un niveau de gravité très élevé, comme dans le cas où ils mènent au suicide. II y a eu un certain nombre d'affaires criminelles contre le

14 Décision n 2-414 / 2012 le 20 février 20, 2012, tribunal du district central de Sotchi (territoire de Krasnodar).

15 Décision n² 2-614 / 20164 juillet 2016, Tribunal de district de Tsimlyansky (région de Rostov).

16 Décision n² 2-638 / 2016 du 8 décembre, 2016, tribunal du district Kirov de Yaroslavl (région de Yaroslavl).

Décision du 25 mai, 2011 Tribunal de district Kirovsky de Rostov-sur-le-Don (région de Rostov). Affaire Krupenko v. Russie. Admiral-leasing LTD.

17 Décision du 25 mai, 2011 Tribunal de district Kirovsky de Rostov-sur-le-Don (région de Rostov). Affaire Krupenko v. Russie. Admiral-leasing LTD. 
personnel militaire ayant harcelé des soldats qui ont commis des suicides ou des tentatives de suicide ${ }^{18}$. II n'y a pas eu de tels exemples en droit du travail. Une autre conclusion, c'est la réticence du juge à considérer le harcèlement comme une discrimination, car les dispositions légales y relatives sont également insuffisantes et plutôt vagues.

\section{C - SANCTIONS DISCIPLINAIRES POUR LES ACTES ASSIMILABLES À DU HARCÈLEMENT}

L'application de sanctions disciplinaires pour les actes de harcèlement au travail pourrait être l'un des piliers de la protection. Cependant, dans trois cas concernant, par exemple, les situations où des salariés ont été sanctionnés pour comportement contraire à l'éthique et pour avoir harcelé leurs collègues, les tribunaux n'ont pas invoqué le principe de protection de la dignité des salariés et hésitent à déclarer que des salariés coupables de harcèlement à l'encontre de leurs collègues avaient commis une infraction disciplinaire si les descriptifs des fonctions de ces salariés ne prévoyaient pas un comportement éthique ${ }^{19}$. Toutefois, si leurs fonctions comprenaient des exigences relatives à la qualité de l'interaction entre collègues, des actes de harcèlement (menaces, insultes, coups) pourraient être jugés contraires au contrat de travail ${ }^{20}$.

\section{II - LA PERTINENCE DE LA CEDH POUR LA PROTECTION DU SALARIÉ CONTRE LE HARCÈLEMENT}

La Convention européenne des droits de l'homme établit les normes qui pourraient être utilisées pour le développement de la protection contre le harcèlement en Russie car, selon la Constitution russe, le droit international est directement applicable par les tribunaux nationaux.

Bien qu'à ce jour, la Cour européenne des droits de l'homme ne se soit jamais prononcée sur la protection des salariés contre le harcèlement ${ }^{21}$, la jurisprudence de la Cour européenne des droits de l'homme sur l'article 3 de la CEDH contient des prémisses importantes pour une telle protection. Ce texte interdit les traitements ou peines dégradants et pourrait être utilisé comme un fondement de la protection des salariés contre le harcèlement au travail.

Cette disposition a été initialement conçue pour offrir une protection contre les traitements dégradants de la part de l'Etat. Mais comme la CEDH est utilisée comme un instrument vivant qui doit être interprété à la lumière des conditions actuelles, la Cour européenne des droits de l'homme s'est réservé une flexibilité suffisante pour traiter de

18 Décision n² 2-826 / 20165, March 30, 2016, Tribunal de district de Khabarovsk.

19 Décision en appel du tribunal de St-Pétersbourg n 33-5330, adoptée le 8 avril 2013 ; Décision du tribunal de Cherkessk n -634/2011, adoptée le 22 mars 2011 ; Décision en appel du tribunal de St-Pétersbourg $n^{\circ}$ 33-6119/2012, adoptée le 17 mai 2012.

20 Décision n 2-484 / 2017, 16 octobre 2017, Tribunal de district industriel (région de Kemerovo).

21 Sauf dans cette affaire où la cour a refusé de conclure à une violation de la CEDH : Dolopoulos v. Grèce (36656/14) 10/12/2015. 
I'application de cet article dans d'autres contextes qui pourraient se présenter ${ }^{22}$. La flexibilité de la Cour européenne des droits de l'homme s'est traduite par le fait qu'elle ait accordé une protection contre les traitements dégradants causés par des personnes privées, par exemple, dans les cas de violence contre les enfants ou de violence domestique ${ }^{23}$.

La notion de traitement inhumain ou dégradant est comprise comme un traitement qui provoque délibérément une souffrance grave, qu'elle soit mentale ou physique ${ }^{24}$. Dans de récentes affaires, la Cour européenne des droits de l'homme a estimé que les souffrances émotionnelles causées par l'humiliation pouvaient constituer une violation de l'article 3 de la $\mathrm{CEDH}^{25}$. Dans l'affaire Đurđević v. Croatie, elle a reconnu la violation de cette disposition en décidant que le harcèlement continu d'une personne handicapée qui a provoqué chez le requérant de la peur et de l'impuissance équivalait à un traitement dégradant ${ }^{26}$. Dans l'affaire Vincent $v$. France, la Cour européenne des droits de l'homme a estimé que le traitement dégradant non intentionnel peut également constituer une violation de la $\mathrm{CEDH}^{27}$. Selon la jurisprudence de la Cour européenne des droits de l'homme, les mauvais traitements doivent atteindre un minimum de gravité pour tomber sous le coup de l'article 3 de la CEDH. L'évaluation de ce minimum est relative : elle dépend de toutes les conditions présentes dans l'affaire, telles que la nature et le contexte du traitement, sa durée et ses effets physiques et mentaux et, dans certains cas, le sexe, l'âge et l'état de la santé de la victime ${ }^{28}$.

Il y a des cas de traitement dégradant qui semblent avoir des points communs avec des situations de harcèlement au travail. Ce dernier provoque de graves souffrances quand la victime est obligée de rester dans un environnement hostile. Dans les cas de harcèlement vertical, la victime est obligée de se plier aux ordres de son bourreau et dépend fortement de lui. Ces caractéristiques du harcèlement présentent des similitudes avec les cas de violence domestique où les victimes sont souvent considérées comme vulnérables, compte tenu de leur dépendance vis-à-vis des auteurs ${ }^{29}$. Ces traits sont également présents dans les situations où les demandeurs se trouvaient dans une position vulnérable du fait qu'ils étaient sous le contrôle d'une autorité, par exemple des militaires enrôlés dans l'armée ${ }^{30}$, qui pouvaient être comparés dans une certaine mesure aux militaires contractuels. Dans ces cas, compte tenu de la vulnérabilité des victimes, la Cour européenne des droits de I'homme réduit la marge d'appréciation laissée à l'Etat et impose une obligation plus stricte de protéger efficacement les droits garantis par l'article 3 de la CEDH.

22 O. De Schutter, "The Protection of Social Rights by the European Court of Human Rights ", in P. Van der Auweraert, T. De Pelsmaeker, J. Sarkin, J. Vande Lanotte, (éd.). Social, Economic and cultural rights. An appraisal of current European and international developments, Anvers, Maklu, 2002, p. 217.

23 ECtHR, A. v. Royaume-Uni (25599/94) 23/09/1998; O'Keeffe v. Irlande (35810/09) 28/01/2014 ; Opuz v. Turquie (33401/02) 09/06/2009.

24 ECtHR, Treholt v. Norvège (14610/89) 09/07/1991; Toth v. Croatie (64674/01) 09/07/2002.

25 ECtHR, R.R. v. Pologne (27617/04) 26/05/2011.

26 ECtHR, Dorđević v. Croatie (41526/10) 24/07/2012.

27 ECtHR, Vincent c. France (6253/03) 24/10/2006.

28 ECtHR, T.M. and C.M. v. République de Moldavie (26608/11) 28/01/2014. Para. 35.

29 ECtHR, Opuz v. Turkey ; Hajduova v. Slovaquie (2660/03) 30/11/ 2010 ; Bevacqua et S. v. Bulgarie (71127/01) 12/06/2008.

30 ECtHR, Placì v. Italie (48754/11) 21/01/2014, Chember v. Russie (7188/03) 03/07/2008. 
Ainsi, la vulnérabilité du requérant pourrait renforcer la revendication de protection contre le traitement dégradant (harcèlement) devant la Cour européenne des droits de l'homme. Selon la jurisprudence de la Cour européenne des droits de l'homme, les Etats ont l'obligation d'assurer une protection efficace contre les traitements dégradants et inhumains, y compris contre la violence d'autres personnes. Par conséquent, l'incapacité des Etats à assurer une protection adéquate contre les traitements inhumains ou dégradants pourrait également constituer une base permettant à la Cour européenne des droits de I'homme de se prononcer dans des affaires de harcèlement moral.

\section{Conclusion}

Cette étude montre que le problème de la protection contre le harcèlement est très aigu en Russie. En l'absence de normes spécifiques, les tribunaux sont réticents à examiner les allégations de harcèlement moral à la lumière du droit du salarié à la dignité. Ils procèdent à l'analyse formelle de la conformité des actes de l'employeur (non-paiement des primes, salaires et modification des conditions de travail, licenciements) avec les règles de procédure établies par le Code du travail russe. Sur une vingtaine environ de cas analysés, il n'y a eu aucun cas de harcèlement moral allégué dans lequel seuls des collègues étaient coupables. L'employeur était toujours l'un des auteurs du harcèlement. En l'absence de législation nationale en la matière, l'approche de la Cour européenne des droits de l'homme face aux actes de traitement dégradant, interdits par l'article 3 de la $\mathrm{CEDH}$, pourrait constituer un argument supplémentaire pour les avocats et renforcer la position des salariés. 